\title{
APPROXIMATION LATTICES DEFINED BY TOLERANCES INDUCED BY IRREDUNDANT COVERINGS
}

\author{
D. GÉGÉNY AND I. PILLER
}

Received 12 March, 2019

\begin{abstract}
The topic of rough set theory considers a relation to determine the lower and upper approximations of a set $X$. Originally, this relation was assumed to be an equivalence relation. This research focuses on using tolerance relations instead of equivalences, i.e. we do not assume the transitivity of the relations. More specifically, in this paper we investigate tolerances induced by irredundant coverings. We characterize the interrelation between the lattices of lower and upper approximations of such tolerances $R$ and $\rho$. The theory of Formal Concept Analysis makes it possible to examine the inclusions of the resulting concepts. We also use quasiorders (denoted by $\unlhd(\rho)$ and $\unrhd(\rho)$ ) and an equivalence relation (denoted by $\operatorname{ker} \rho$ ) for summarizing the connection between tolerances and lattices in a theorem.
\end{abstract}

2010 Mathematics Subject Classification: 06B15; 68T37; 06B23

Keywords: rough set, tolerance relation, irredundant coverings

\section{INTRODUCTION}

The notion of rough sets was introduced by Z. Pawlak [8]. His idea was that our knowledge about the elements of a universe $U$ is given in terms of an information relation $R \subseteq U \times U$ reflecting their indiscernibility. Originally, Pawlak assumed that this binary relation is an equivalence, but later several other types of relations were also examined (see e.g. [4,12], or [6]). For any binary relation $R \subseteq U \times U$ and any element $u \in U$, denote by $R(u)$ the $R$-neighbourhood of $u$, i.e. $R(u):=\{x \in U \mid$ $(u, x) \in R\}$. Now, for any subset $X \subseteq U$ the lower approximation of $X$ is defined as

$$
X_{R}:=\{x \in U \mid R(x) \subseteq U\},
$$

and the upper approximation of $X$ is given by

$$
X^{R}:=\{x \in U \mid R(x) \cap X \neq \varnothing\} .
$$

If $R$ is a reflexive relation then $X_{R} \subseteq X \subseteq X^{R}$. The rough set of $X$ is the pair $\left(X_{R}, X^{R}\right)$, and the set of all rough sets is

$$
R S(U, R)=\left\{\left(X_{R}, X^{R}\right) \mid X \subseteq U\right\} .
$$

(c) 2019 Miskolc University Press 
The set $R S(U, R)$ may be canonically ordered by the component-wise inclusion:

$$
\left(X_{R}, X^{R}\right) \leq\left(Y_{R}, Y^{R}\right) \Longleftrightarrow X_{R} \subseteq Y_{R} \text { and } X^{R} \subseteq Y^{R},
$$

obtaining a partially ordered $\operatorname{set} \mathbf{R S}(U, R):=(R S(U, R), \leq)$. If $R$ is an equivalence, then $\mathbf{R S}(U, R)$ is a particular complete distributive lattice.

Ordering the sets $\wp(U)^{R}=\left\{X^{R} \mid X \subseteq U\right\}$ and $\wp(U)_{R}=\left\{X_{R} \mid X \subseteq U\right\}$ by the relation $\subseteq$ we obtain dually isomorphic complete lattices $\left(\wp(U)^{R}, \subseteq\right)$ and $\left(\wp(U)_{R}, \subseteq\right)$, called the lattice of upper approximations, respectively the lattice of lower approximations (see [6]). Let $R$ be a tolerance, that is, a reflexive and symmetric relation. In [6] it was shown that $\left(\wp(U)_{R}, \subseteq\right)$ is isomorphic to the concept lattice of the context $\left(U, U, R^{c}\right)$, where $R^{c}=(U \times U) \backslash R$ is the complement of the relation $R$. By using this observation, in [2], we applied FCA methods to describe the sublattices of the lattices of upper (lower) approximations. These lattices play an important role in several applications of rough set theory (see $[3,9-11,13])$.

This paper can be considered as a continuation of [2] where we deduced sufficient conditions which guarantee that for some tolerances $R \subseteq \rho \subseteq U \times U$, the lattice $\wp(U)^{\rho}\left(\wp(U)_{\rho}\right)$ is a complete sublattice of $\wp(U)^{R}$ (of $\left.\wp(U)_{R}\right)$. The focus of this paper is on the approximation lattices defined by tolerances induced by irredundant coverings of $U$. These relations can be considered as a natural generalization of equivalences. If $R \subseteq \rho \subseteq U \times U$ are tolerance relations and $R$ is induced by an irredundant covering of $U$, we characterize the case when the concept lattice $\mathscr{L}\left(U, U, \rho^{c}\right)$ is a complete sublattice of the concept lattice $\mathscr{L}\left(U, U, R^{c}\right)$. Then this characterization is applied to compare the lattices $\left(\wp(U)^{R}, \subseteq\right)$ and $\left(\wp(U)_{R}, \subseteq\right)$.

\section{PRELIMINARIES}

First, we note that the above defined approximations for any $X \subseteq U$ and any $\mathscr{H} \subseteq$ $\mathcal{P}(U)$ have the following properties:

$$
\begin{aligned}
& \text { (a) }\left(\bigcup_{X \in \mathscr{H}} X\right)^{R}=\bigcup_{X \in \mathscr{H}} X^{R} \text { and }\left(\bigcap_{X \in \mathscr{H}} X\right)_{R}=\bigcap_{X \in \mathscr{H}} X_{R} \text {; } \\
& \text { (b) }\left(X^{c}\right)^{R}=\left(X_{R}\right)^{c},\left(X^{c}\right)_{R}=\left(X^{R}\right)^{c} .
\end{aligned}
$$

In view of $(\mathrm{a}), \wp(U)_{R}$ is a closure system, being closed under arbitrary intersections and $\wp(U)^{R}$ is an interior system, because it is closed under any union. Therefore, $\wp(U)_{R}$ and $\wp(U)^{R}$ are complete lattices with respect to $\subseteq$. If $R$ is a tolerance relation, then for any $X, Y \subseteq U$ we have: $X^{R} \subseteq Y \Leftrightarrow X \subseteq Y_{R}$.

Property (b) implies that the lattices $\left(\wp(U)_{R}, \subseteq\right)$ and $\left(\wp(U)^{R}, \subseteq\right)$ are dually isomorphic via the map $H: \wp(U)_{R} \rightarrow \wp(U)^{R}, H: X \rightarrow X^{c}$, since $H\left(X_{R}\right)=\left(X_{R}\right)^{c}=$ $\left(X^{c}\right)^{R}$. If $R$ is an equivalence, then $\wp(U)_{R}=\wp(U)^{R}$ and they form the same Boolean lattice.

A formal context is a triple $\mathcal{K}=(G, M, I)$, where $G$ is a set of objects, $M$ is a set of attributes and $I \subseteq G \times M$ is a relation, called incidence relation. The notations 
$(g, m) \in I$ and $g I m$ both express that an object $g$ is in relation $I$ with an attribute $m$. The basics of Formal Concept Analysis (FCA) can be found e.g. in [1]. By defining for all subsets $A \subseteq G$ and $B \subseteq M$

$$
\begin{aligned}
& A^{I}=\{m \in M \mid(g, m) \in I, \text { for all } g \in A\}, \\
& B^{I}=\{g \in G \mid(g, m) \in I, \text { for all } m \in B\}
\end{aligned}
$$

we establish a Galois connection between the power-set lattices $\wp(G)$ and $\wp(M)$ and the maps $A \rightarrow A^{I I}, A \subseteq G$ and $B \rightarrow B^{I I}, B \subseteq M$ are closure operators on $\wp(G)$, respectively $\wp(M)$.

A formal concept of the context $\mathcal{K}$ is a pair $(A, B) \in \wp(G) \times \wp(M)$ with $A^{I}=B$ and $B^{I}=A$, where the set $A$ is called the extent and $B$ is called the intent of the concept $(A, B)$. It is easy to check that a pair $(A, B) \in \wp(G) \times \wp(M)$ is a concept if and only if $(A, B)=\left(A^{I I}, A^{I}\right)=\left(B^{I}, B^{I I}\right)$. The set of all concepts of the context $\mathcal{K}$ is denoted by $\mathscr{L}(\mathcal{K})$. This set $\mathscr{L}(\mathcal{K})$ is ordered by

$$
\left(A_{1}, B_{1}\right) \leq\left(A_{2}, B_{2}\right) \Leftrightarrow A_{1} \subseteq A_{2} \Leftrightarrow B_{1} \supseteq B_{2} .
$$

With respect to this order, $\mathscr{L}(\mathcal{K})$ forms a complete lattice, called the concept lattice of the context $\mathcal{K}=(G, M, I)$, denoted by $\mathscr{L}(G, M, I)$.

A relation $J \subseteq I$ is called a closed subrelation of the context $(G, M, I)$ if every concept of the context $(G, M, J)$ is also a concept of $(G, M, I)$. In [1] it is proved that this definition is equivalent to the condition that the concept lattice $\mathscr{L}(G, M, J)$ is a complete sublattice of $\mathscr{L}(G, M, I)$.

For a tolerance relation $R \subseteq U \times U$ the relationship between the lattices of approximations and the concept lattice $\mathscr{L}\left(U, U, R^{c}\right)$ was described in [6]. Indeed, let $I=R^{c}$. Then for any $X \subseteq U$ we have

$$
\begin{aligned}
X^{I} & =\left\{u \in U \mid x R^{c} u, \text { for all } x \in X\right\} \\
& =\{u \in U \mid(x, u) \notin R, \text { for all } x \in X\}=U \backslash X^{R}=\left(X^{R}\right)^{c} .
\end{aligned}
$$

Thus $X^{R}=\left(X^{I}\right)^{c}$, and $X_{R}=\left(\left(X^{c}\right)^{R}\right)^{c}=\left(X^{c}\right)^{I}$, according to (b).

In [6] it is also proved that $\left(\wp(U)^{R}, \subseteq\right) \cong\left(\wp(U)_{R}, \supseteq\right) \cong \mathscr{L}\left(U, U, R^{c}\right)$.

\section{COMPLETE SUBLATTICES OF APPROXIMATION LATTICES}

Now let $\rho, R$ be two tolerance relations such that $R \subseteq \rho \subseteq U \times U$. Consider the formal contexts $\mathcal{K}_{R}=\left(U, U, R^{c}\right)$ and $\mathcal{K}_{\rho}=\left(U, U, \rho^{c}\right)$. Since $J:=\rho^{c} \subseteq R^{c}:=I$, $\mathcal{K}_{\rho}$ is a subcontext of $\mathcal{K}_{R}$. We intend to characterize the case when the lattice $\wp(U)^{\rho}\left(\wp(U)_{\rho}\right)$ is isomorphic (dually isomorphic) to a complete sublattice of $\wp(U)^{R}$ $\left(\wp(U)_{R}\right.$, respectively). In [2] we proved that $\left(\wp(U)^{\rho}, \subseteq\right)$ is a complete sublattice of $\left(\wp(U)^{R}, \subseteq\right)$, respectively $\left(\wp(U)_{\rho}, \subseteq\right)$ is a complete sublattice of $\left(\wp(U)_{R}, \subseteq\right)$, whenever $\mathscr{L}\left(U, U, \rho^{c}\right)$ is a complete sublattice of $\mathscr{L}\left(U, U, R^{c}\right)$. Unfortunately, the 
converse implication does not necessarily hold. For instance, in [2] we constructed an example where $\left(\wp(U)^{\rho}, \subseteq\right)$ is a complete sublattice of $\left(\wp(U)^{R}, \subseteq\right)$, however $\mathscr{L}\left(U, U, \rho^{c}\right)$ is not even a subset of the lattice $\mathscr{L}\left(U, U, R^{c}\right)$. All we can say is that in general the following conditions are equivalent:

(1) $\left(\wp(U)^{R}, \subseteq\right)$ is isomorphic to a complete sublattice of $\left(\wp(U)^{\rho}, \subseteq\right)$;

(2) $\left(\wp(U)_{R}, \subseteq\right)$ is isomorphic to a complete sublattice of $\left(\wp(U)_{\rho}, \subseteq\right)$;

(3) $\mathscr{L}\left(U, U, \rho^{c}\right)$ is isomorphic to a complete sublattice of $\mathscr{L}\left(U, U, R^{c}\right)$.

Let $\rho$ be a tolerance on $U$. Let us define

$$
\begin{aligned}
\unlhd(\rho) & :=\{(x, y) \in U \times U \mid \rho(x) \subseteq \rho(y)\}, \\
\unrhd(\rho) & :=\{(x, y) \in U \times U \mid \rho(x) \supseteq \rho(y)\}, \\
\operatorname{ker} \rho & :=\{(x, y) \in U \times U \mid \rho(x)=\rho(y)\} .
\end{aligned}
$$

Clearly, $\unlhd(\rho)$ and $\unrhd(\rho)$ are reflexive and transitive relations, i.e. they are quasiorders and $\unrhd(\rho)$ is the inverse relation of $\unlhd(\rho)$. $\operatorname{ker} \rho$ is an equivalence relation, called the kernel of the tolerance $\rho$. Clearly, $\operatorname{ker} \rho=\unlhd(\rho) \cap \unrhd(\rho)$. Let the symbol $\circ$ stand for the relational product, in what follows. It is easy to check that in the case $R \subseteq \rho$ the relations $R \circ \unlhd(\rho) \subseteq \rho$ and $\unrhd(\rho) \circ R \subseteq \rho$ always hold. Using these notions, in [2] we proved the following characterization:

Theorem 1. Let $\rho, R$ be two tolerance relations satisfying $R \subseteq \rho \subseteq U \times U$. Then the following conditions are equivalent:

(C): $\mathscr{L}\left(U, U, \rho^{c}\right)$ is a complete sublattice of $\mathscr{L}\left(U, U, R^{c}\right)$;

(D): For any $(a, b) \in \rho \backslash R$ there exist some elements $c, d \in U$ such that $(b, c),(a, d) \in R$ and $\rho(c) \subseteq \rho(a), \rho(d) \subseteq \rho(b) ;$

(E): $R \circ \unlhd(\rho)=\rho$;

$\left(\mathbf{E}^{\prime}\right): \unrhd(\rho) \circ R=\rho$.

Now, the next corollary is immediate:

Corollary 1. Let $R, \rho$ be two tolerance relations on $U$ such that $R \subseteq \rho$. If $R$ and $\rho$ satisfy one of the equivalent conditions of Theorem 1 , then $\left(\wp(U)^{\rho}, \subseteq\right)$ is a complete sublattice of $\left(\wp(U)^{R}, \subseteq\right)$ and $\left(\wp(U)_{\rho}, \subseteq\right)$ is a complete sublattice of $\left(\wp(U)_{R}, \subseteq\right)$.

In [2] we proved that in the particular case when $\rho$ is an equivalence relation on $U$ such that $R \subseteq \rho$, then condition (D) is satisfied. Hence in such a case $\left(\wp(U)^{\rho}, \subseteq\right)$ is obviously a sublattice of $\left(\wp(U)^{R}, \subseteq\right)$ and $\left(\wp(U)_{R}, \subseteq\right)$. 
Here we give an algorithm (Algorithm 1) that is checking on a finite set $U$ and two relations $R \subseteq \rho \subseteq U \times U$ whether $\mathscr{L}\left(U, U, \rho^{c}\right)$ is a complete sublattice of $\mathscr{L}\left(U, U, R^{c}\right)$ by using condition (D).

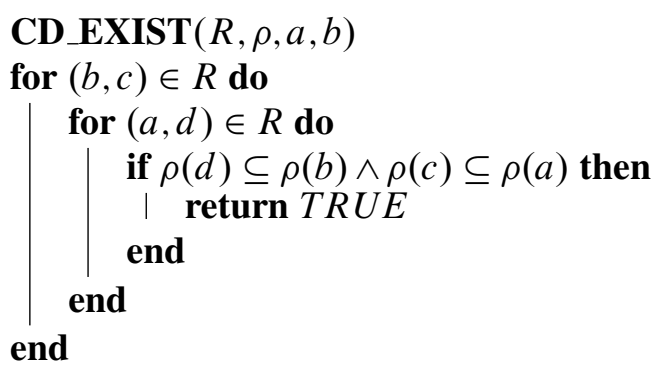

return FALSE

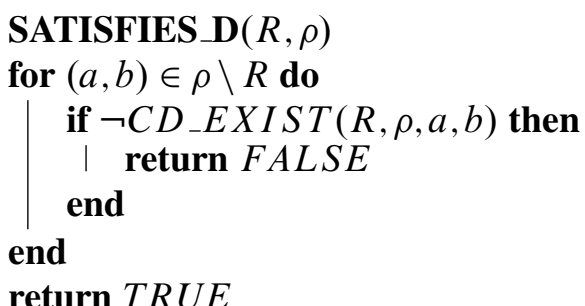

Algorithm 1: Algorithm for checking condition (D)

\section{TOLERANCES INDUCED BY IRREDUNDANT COVERINGS}

A collection $\mathcal{C}$ of nonempty subsets of $U$ is called a covering of $U$ if $\cup \mathcal{C}=U$. The covering $\mathcal{C}$ is called irredundant if removing any member $X$ of $\mathcal{C}$, the collection

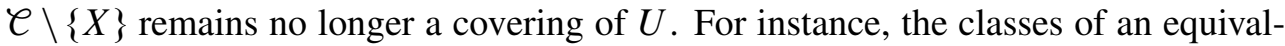
ence relation $E \subseteq U \times U$ provide a simple example of an irredundant covering of $U$. Each covering $\mathscr{C}$ of $U$ defines a tolerance relation $\rho \mathcal{C}=\bigcup\{X \times X \mid X \in \mathcal{C}\}$, called the tolerance induced by $\mathcal{C}$. If $\mathcal{C}$ is an irredundant covering of $U$, then we say that $\rho e$ is a tolerance induced by an irredundant covering. In [6] the authors proved that the lattices $\wp(U)^{\rho}, \wp(U)_{\rho}$ and $\mathbf{R S}(U, \rho)$ are completely distributive if and only if $\rho$ is induced by an irredundant covering of $U$. It was shown that this condition is also equivalent to the condition that the lattice $\mathscr{L}\left(U, U, \rho^{c}\right)$ is completely distributive. A complete lattice $L$ is called completely distributive (see e.g. [1]) if for any doubly indexed family of elements $\left\{x_{i, j}\right\}_{i \in I, j \in J},(I, J \neq \varnothing)$ we have

$$
\bigwedge_{i \in I}\left(\bigvee_{j \in J} x_{i, j}\right)=\bigvee_{f: I \rightarrow J}\left(\bigwedge_{i \in I} x_{i, f(i)}\right)
$$


We note that any complete sublattice of a completely distributive lattice is also completely distributive. As immediate consequence of the mentioned results we obtain the following Lemma.

Lemma 1. Let $R, \rho$ be two tolerance relations on $U$ with $R \subseteq \rho$ and such that $\left(\wp(U)^{R}, \subseteq\right)$ is isomorphic to a complete sublattice of $\left(\wp(U)^{\rho}, \subseteq\right)$. If $R$ is a tolerance induced by an irredundant covering, then $\rho$ is also a tolerance induced by an irredundant covering.

Proof. Since in view of [6] $\left(\wp(U)^{R}, \subseteq\right)$ is a completely distributive lattice, $\left(\wp(U)^{\rho}, \subseteq\right)$, being a complete sublattice of $\left(\wp(U)^{R}, \subseteq\right)$, is also completely distributive. Therefore, $\rho$ is a tolerance induced by an irredundant covering.

Since any equivalence relation is a particular tolerance induced by an irredundant covering, the following corollary is immediate.

Corollary 2. Let $R$ be an equivalence relation and $\rho$ a tolerance relation on $U$ with $R \subseteq \rho$ and such $\left(\wp(U)^{R}, \subseteq\right)$ is a complete sublattice of $\left(\wp(U)^{\rho}, \subseteq\right)$. Then $\rho$ is induced by an irredundant covering.

Let $\rho$ be a tolerance on $U$. A nonempty subset $X$ of $U$ is called a preblock of $\rho$ if $X \times X \subseteq \rho$. Note that in this case $B \subseteq \rho(x)$ for all $x \in B$. A preblock of $\rho$ that is maximal with respect to the inclusion is called a block of $\rho$.

Remark 1. It is well known that any tolerance relation $\rho$ is determined by its blocks, that is for any $a, b \in U,(a, b) \in \rho \Leftrightarrow a, b \in B$, for some block $B$ of $\rho$. In [6] and in [7] it is shown that if $\rho$ is induced by an irredundant covering $\mathcal{C}$, then $B$ can be chosen as a member of $\mathcal{C}$ having the property $B=\rho(k)$, for some $k \in B$. It is also proved that in this case $\rho=\unrhd(\rho) \circ \unlhd(\rho)$ (see [5]).

Theorem 2. Let $\rho, R \subseteq U \times U$ be two tolerance relations with $R \subseteq \rho$ and assume that $R$ is induced by an irredundant covering. Then condition $(E)$ is equivalent to the condition

(F): $\rho=(R \cap \unrhd(\rho)) \circ \operatorname{ker} \rho \circ(R \cap \unlhd(\rho))$.

Proof. First we will show that condition (E) implies condition (F). If (E) holds, then $\mathscr{L}\left(U, U, \rho^{c}\right)$ is a complete sublattice of $\mathscr{L}\left(U, U, R^{c}\right)$, according to Theorem 1 . By Corollary 1 this yields that $\left(\wp(U)^{\rho}, \subseteq\right)$ is a complete sublattice of $\left(\wp(U)^{R}, \subseteq\right)$. Then by Lemma $1, \rho$ is also a tolerance induced by an irredundant covering of $U$. Then $\rho=\unrhd(\rho) \circ \unlhd(\rho)$, according to [5]. It is easy to check that $\unrhd(\rho) \circ \operatorname{ker} \rho \subseteq \unrhd(\rho)$. Hence

$(R \cap \unrhd(\rho)) \circ \operatorname{ker} \rho \circ(R \cap \unlhd(\rho)) \subseteq \unrhd(\rho) \circ \operatorname{ker} \rho \circ \unlhd(\rho) \subseteq \unrhd(\rho) \circ \unlhd(\rho)=\rho$.

In order to prove the converse inclusion, take any $(a, b) \in \rho$. Then, in view of Remark 1 , there exists a $k \in U$ and a block $B$ of $\rho$ such that $a, b \in B=\rho(k)$. Then $B \subseteq \rho(a), \rho(b)$. As $(a, k),(b, k) \in \rho$, now condition (E), i.e. $R \circ \unlhd(\rho)=\rho$ 
implies that there exist some elements $c, d \in U$ such that $(a, c) \in R, \rho(c) \subseteq \rho(k)$ and $(b, d) \in R, \rho(d) \subseteq \rho(k)$. Since $c, d \in \rho(k)=B$, we have $B \subseteq \rho(c), \rho(d)$, whence we get $\rho(c)=\rho(d)=B$, proving $(c, d) \in \operatorname{ker} \rho$. Then $\rho(c)=B \subseteq \rho(a)$ also yields $(a, c) \in \unrhd(\rho)$. Hence $(a, c) \in R \cap \unrhd(\rho)$. Similarly, $\rho(d)=B \subseteq \rho(b)$ yields $(d, b) \in \unlhd(\rho)$. Thus $(d, b) \in R \cap \unlhd(\rho)$. Now, $(a, c) \in R \cap \unrhd(\rho),(c, d) \in \operatorname{ker} \rho$ and $(d, b) \in R \cap \unlhd(\rho)$ together imply $(a, b) \in(R \cap \unrhd(\rho)) \circ \operatorname{ker} \rho \circ(R \cap \unlhd(\rho))$, proving $\rho \subseteq(R \cap \unrhd(\rho)) \circ \operatorname{ker} \rho \circ(R \cap \unlhd(\rho))$.

Conversely, assume that $(\mathrm{F})$ holds, i.e. $\rho=(R \cap \unrhd(\rho)) \circ \operatorname{ker} \rho \circ(R \cap \unlhd(\rho))$. We will prove (E'), which is equivalent to (E) by Theorem 1. Since $\unrhd(\rho) \circ R \subseteq \rho$ is always true, we have to show only the converse inclusion. Indeed, take any $(a, b) \in \unrhd$ $(\rho) \circ R$. Then there exist some elements $d, c \in U$ such that $(a, c) \in R \cap \unrhd(\rho),(c, d) \in$ $\operatorname{ker} \rho$, and $(d, b) \in R \cap \unlhd(\rho)$. Hence $\rho(c)=\rho(d)$. As $(a, c) \in \unrhd(\rho)$ means that $\rho(c) \subseteq \rho(a)$, we get also $\rho(d) \subseteq \rho(a)$, i.e. $(a, d) \in \unrhd(\rho)$. Since $(d, b) \in R$, we obtain $(a, b) \in \unrhd(\rho) \circ R$. Hence $\rho \subseteq \unrhd(\rho) \circ R$, and this means that condition (E') is satisfied.
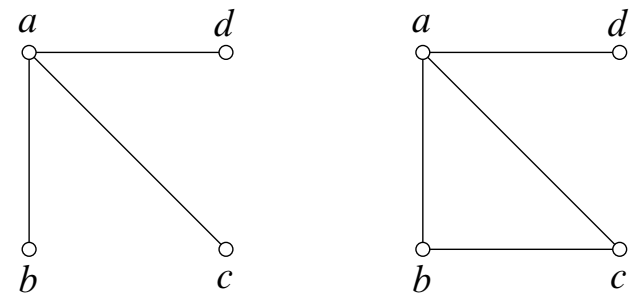

FIGURE 1. Relations $R$ and $\rho$ satisfying condition (F)

TABLE 1 . The contexts $\left(U, U, R^{c}\right)$ and $\left(U, U, \rho^{c}\right)$

\begin{tabular}{c|c|c|c|c}
$R^{c}$ & $a$ & $b$ & $c$ & $d$ \\
\hline$a$ & & & & \\
\hline$b$ & & & $\times$ & $\times$ \\
\hline$c$ & & $\times$ & & $\times$ \\
\hline$d$ & & $\times$ & $\times$ & \\
\hline
\end{tabular}

\begin{tabular}{c|c|c|c|c}
$\rho^{c}$ & $a$ & $b$ & $c$ & $d$ \\
\hline$a$ & & & & \\
\hline$b$ & & & & $\times$ \\
\hline$c$ & & & & $\times$ \\
\hline$d$ & & $\times$ & $\times$ & \\
\hline
\end{tabular}

Corollary 3. Let $R, \rho$ be two tolerance relations on $U$ such that $R \subseteq \rho$. If $R$ and $\rho$ satisfy condition $(F)$ and $R$ is induced by an irredundant covering of $U$ then $\left(\wp(U)^{\rho}, \subseteq\right)$ is a complete sublattice of $\left(\wp(U)^{R}, \subseteq\right)$ and $\left(\wp(U)_{\rho}, \subseteq\right)$ is a complete sublattice of $\left(\wp(U)_{R}, \subseteq\right)$.

Corollary 4. Let $R, \rho$ be two tolerance relations on $U$ such that $R \subseteq \rho$, condition $(F)$ holds and $R \subseteq \operatorname{ker} \rho$. Then $\rho$ is an equivalence. 
Proof. If $R \subseteq \operatorname{ker} \rho$ then $R \cap \unrhd(\rho) \subseteq \operatorname{ker} \rho$ and $R \cap \unlhd(\rho) \subseteq \operatorname{ker} \rho$. This implies that $(R \cap \unrhd(\rho)) \circ \operatorname{ker} \rho \circ(R \cap \unlhd(\rho)) \subseteq \operatorname{ker} \rho \circ \operatorname{ker} \rho \circ \operatorname{ker} \rho=\operatorname{ker} \rho$, since $\operatorname{ker} \rho$ is an equivalence. Thus, we get $\rho \subseteq \operatorname{ker} \rho$ using condition $(F)$. Since $\rho$ is a tolerance relation, $\operatorname{ker} \rho \subseteq \rho$ also holds, meaning $\rho=\operatorname{ker} \rho$, therefore $\rho$ is an equivalence.
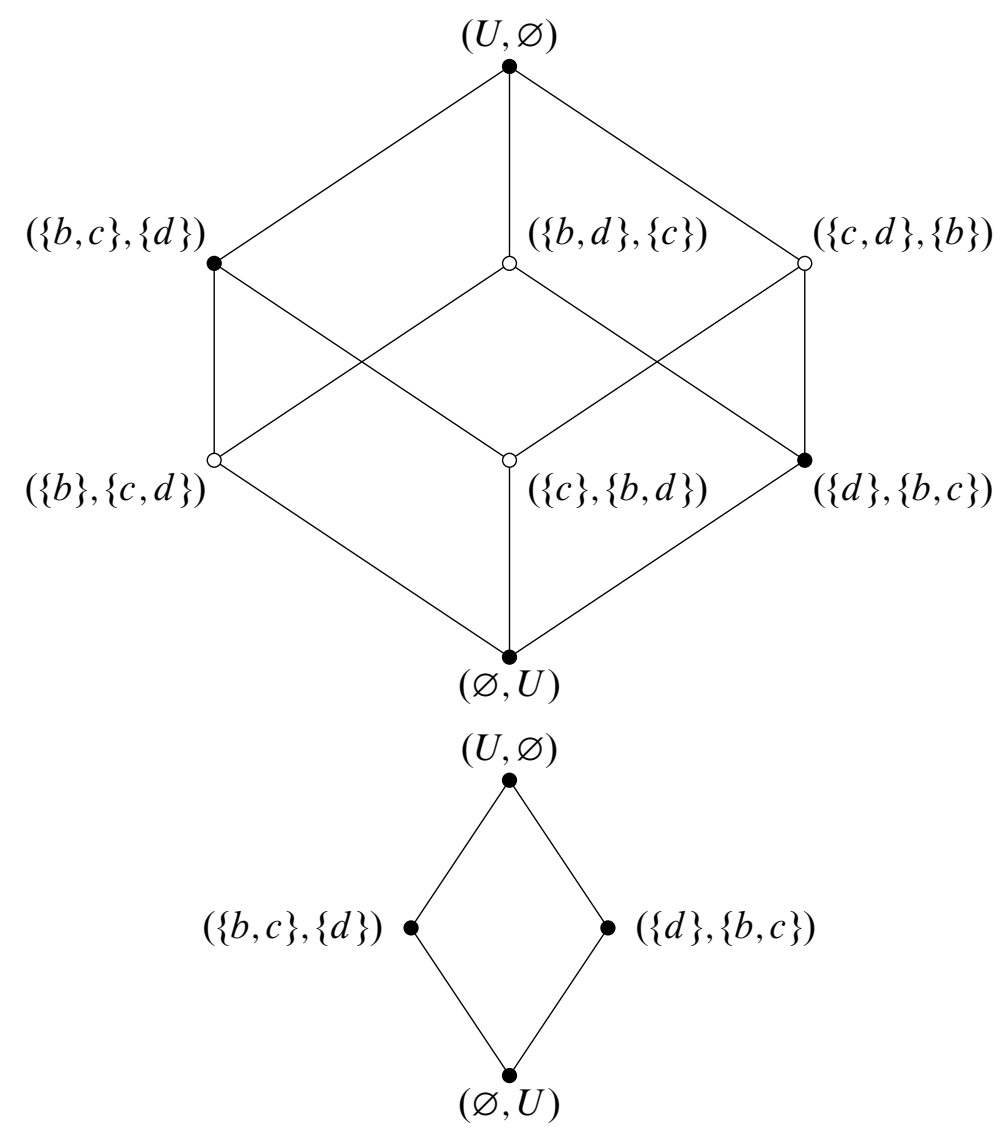

FIgURE 2. The Hasse-diagrams of the concept lattices $\mathscr{L}\left(U, U, R^{c}\right)$ and $\mathscr{L}\left(U, U, \rho^{c}\right)$

Corollary 5. Let $R, \rho$ be two tolerance relations on $U$ such that $R \subseteq \rho$. If $\rho$ is an equivalence, condition $(F)$ automatically holds.

Proof. Since $\rho$ is an equivalence, $\rho=\operatorname{ker} \rho=\unrhd(\rho)=\unlhd(\rho)$. Therefore $R \cap \unrhd$ $(\rho)=R \cap \unlhd(\rho)=R \cap \rho=R$. Then it follows that $(R \cap \unrhd(\rho)) \circ \operatorname{ker} \rho \circ(R \cap \unlhd(\rho))$ $=R \circ \rho \circ R$. However, $R \circ \rho \circ R \subseteq \rho \circ \rho \circ \rho=\rho$. On the other hand, $R \circ \rho \circ R \supseteq$ $\Delta \circ \rho \circ \Delta=\rho$, where $\Delta$ is the identity relation. Combining them yields $R \circ \rho \circ R=\rho$, which proves condition $(F)$. 


\section{CONCLUSION}

In this paper, we were aiming to extend the characterization found in [2] by further investigating the tolerance relations $R \subseteq \rho$. We deduced condition (F), which is equivalent to the conditions in Theorem 1 whenever $R$ is a tolerance induced by an irredundant covering. Additionally, an algorithm for checking condition (D) was also provided. An example of two relations satisfying condition (F) can be seen in Figure 1. Since tolerance relations are always reflexive, loops are not noted on the figure for simplicity. Figure 2 shows that the concept lattice $\mathscr{L}\left(U, U, \rho^{c}\right)$ is a complete sublattice of $\mathscr{L}\left(U, U, R^{c}\right)$, i.e. condition (C) holds. We also proved some consequences for special cases, e.g. $R$ being a subrelation of $\operatorname{ker} \rho$. As a future work, we propose investigating the results in [2] regarding the so-called compatibility condition in combination with tolerances induced by an irredundant covering.

\section{REFERENCES}

[1] B. Ganter and R. Wille, Formal concept analysis: mathematical foundations. Springer Science \& Business Media, 2012.

[2] D. Gégény, I. Piller, S. Radeleczki, and L. Veres, "Approximations induced by tolerance relations," 2019.

[3] J. Järvinen, L. Kovács, and S. Radeleczki, "Defining rough sets using tolerances compatible with an equivalence," 2019.

[4] J. Järvinen, P. Pagliani, and S. Radeleczki, "Information completeness in Nelson algebras of rough sets induced by quasiorders," Studia logica, vol. 101, no. 5, pp. 1073-1092, 2013, doi: 10.1007/s11225-012-9421-z.

[5] J. Järvinen and S. Radeleczki, "Monteiro spaces and rough sets determined by quasiorder relations: models for Nelson algebras," Fundamenta Informaticae, vol. 131, no. 2, pp. 205-215, 2014.

[6] J. Järvinen and S. Radeleczki, "Rough sets determined by tolerances," International Journal of Approximate Reasoning, vol. 55, no. 6, pp. 1419-1438, 2014, doi: 10.1016/j.ijar.2013.12.005.

[7] J. Järvinen and S. Radeleczki, "Irredundant coverings, tolerances, and related algebras," in Algebraic Methods in General Rough Sets. Springer, 2018, pp. 417-457, doi: 10.1007/978-3-03001162-8'5.

[8] Z. Pawlak, "Rough sets," International journal of computer \& information sciences, vol. 11, no. 5, pp. 341-356, 1982, doi: 10.1007/BF01001956.

[9] Z. Pawlak, "Rough set theory and its applications to data analysis," Cybernetics \& Systems, vol. 29, no. 7, pp. 661-688, 1998, doi: 10.1080/019697298125470.

[10] J. A. Pomykala, "On similarity based approximation of information," Demonstratio Mathematica, vol. 27, no. 3-4, pp. 663-671, 1994, doi: 10.1515/dema-1994-3-412.

[11] S. Rissino and G. Lambert-Torres, "Rough set theory-fundamental concepts, principals, data extraction, and applications," in Data mining and knowledge discovery in real life applications. InTech, 2009. doi: 10.5772/6440.

[12] Y. Yao, "Generalized rough set models," Rough sets in knowledge discovery, vol. 1, pp. 286-318, 1998.

[13] W. Zakowski, "Approximations in the space $(u, \pi)$," Demonstratio mathematica, vol. 16, no. 3, pp. 761-770, 1983, doi: 10.1515/dema-1983-0319. 
Authors' addresses

D. Gégény

University of Miskolc, Institute of Mathematics, H-3515 Miskolc-Egyetemváros, Hungary

E-mail address: matgdeuni-miskolc.hu

I. Piller

University of Miskolc, Institute of Mathematics, H-3515 Miskolc-Egyetemváros, Hungary

E-mail address: imre.piller@uni-miskolc.hu 